\title{
Frequency Upshifting and Pulse Compression via Underdense Relativistic Ionization Fronts
}

\author{
R. L. Savage, Jr., R. P. Brogle, W. B. Mori, and C. Joshi, Fellow, IEEE
}

(Invited Paper)

\begin{abstract}
When pulse of electromagnetic radiation impinges upon a moving plasma/neutral gas boundary, i.e., an ionization front, its frequency and duration are altered. The largest frequency upshifts and pulse compressions are obtained when radiation is incident on an ionization front moving at close to the speed of light. Such an ionization front is created when a short laser pulse photo-ionizes a gas through which it propagates. We have done theoretical and experimental work on the interaction of radiation with a relativistically propagating, underdense ionization front in a waveguide. In the experiment, $35 \mathrm{GHz}$ microwave pulses were upshifted and compressed upon encountering a moving front. The frequency spectrum of the upshifted radiation was determined independently using sections of cutoff waveguides and a microwave diffraction grating. These frequency upshifts were proportional to the plasma density of the ionization front as predicted by the theory. The front density was determined using microwave interferometery. The pusewidths of the upshifted radiation were measured with fast diode detectors. These pulsewidth measurements were also in good agreement with the theory. Frequency upshifts and pulse compressions up to a factor of five were recorded in this experiment.
\end{abstract}

\section{INTRODUCTION}

$\mathbf{W}$ HEN a pulse of electromagnetic radiation reflects from a moving mirror, both its frequency and its duration are altered by the relativistic Doppler effect [1] which depends strongly on the velocity of the mirror. It has been predicted that similar changes would occur if the radiation were to reflect from a moving plasma/neutral gas boundary, and ionization front [2], [3]. An ionization front is created, for instance, when an ionizing laser pulse passes through a region of neutral gas. The Doppler shift is the same in both cases, but unlike the mirror, the ionzation front has no kinetic energy. Therefore, in contrast to the moving mirror, the number of photons is not conserved upon reflection from the front, and the energy in the reflected pulse may be much less than the incident pulse energy. In order to reflect the impinging radiation, the front must be overdense; that is, the density of the plasma in the front must be above the critical density for electromagnetic radiation when viewed from the rest frame of the front. The critical density is defined as that density for which the characteristic plasma frequency, $\omega_{p}$, equals the

Manuscript received October 15,1992 . This work was supported by the U.S. Department of Energy Grant DE-FG03-91-ER12114.

The authors are with the Department of Electrical Engineering and Physics, UCLA, Los Angeles, CA 90024.

IEEE Log Number 9206338. radiation frequency. The plasma frequency is defined by

$$
\omega_{p}=\left[\frac{4 \pi n_{0} e^{2}}{m}\right]^{1 / 2}
$$

where $n_{0}$ is the plasma density, $m$ is the electrons's mass, and $e$ is its charge. Because the frequency of the incident radiation is higher when viewed from this frame and the plasma frequency is Lorentz invariant, creation of an overdense front requires that the plasma be more dense than for a stationary boundary. Thus creation of an overdense front is often technologically impracticable. However, it has recently been predicted that large frequency upshifts and pulse compressions are possible even for underdense relativistic fronts, where the incident radiation is transmitted into the plasma [4]. In this case the degree of frequency upshift is proportional to the plasma density, and unlike reflection from an overdense front, is relatively insensitive to the front's velocity.

Developments in laser technology have enabled the generation of relativistically propagating ionization fronts by intense short-pulse lasers via photo-ionization. Several groups have reported that either a portion of the ionizing radiation or a separate probe pulse was blue-shifted as it copropagated with the front [5]-[7]. This upshifting results from phase modulation due to the rapid formation of the plasma as the radiation propagates with the density gradient at the plasma/neutral gas boundary. Novel radiation sources utilizing similar copropagating ionization fronts or relativistic plasma waves have been proposed [8]-[10]. To obtain large upshifts, these schemes require that the upshifting radiation and the density gradient copropagate over a considerable distance. Conversely, in a geometry in which radiation impinges upon the front, the radiation and the front do not propagate at the same velocity. The ultimate upshift in this case depends only on the maximum density in the front, and is acquired in a relatively short interaction region [11]-[13]. Furthermore, for large upshifts the incident pulse is significantly reduced in duration [4], as in the case for reflection from overdense fronts [3], [14].

Using a fixed-frequency source for the incident radiation and an ionizing laser with a fixed output, one can create a tunable source of upshifted radiation by simply varying the neutral gas density in the interaction region. The frequency of the upshifted radiation can be continuously tuned to many times the source frequency. Furthermore, because the upshifted pulse is generated only during the transit time of the laser pulse through the interaction region (and may be compressed 
to much less than the transit time), the upshifted pulse can be very short in duration even if the source gives much longer output pulses or even continuous output. Tunable, short-pulse radiation sources derived from this technique have potential applications in many areas, including plasma diagnostics, spectroscopy, and remote sensing.

The goal of this work was to test the predictions of the underdense ionization front theory, and in doing so to create a new type of tunable radiation source. Our experiments utilize a fixed-frequency source of microwave radiation operating at approximately $35 \mathrm{GHz}$. The front is created by a 50 pseclong ionizing laser pulse. The interaction occurs inside a metallic waveguide where the group velocity of the source radiation is about half of the free space value, $c$. The metallic waveguide is used to reduce the transverse spatial extent of the source wave. Reducing the volume of the interaction region allows one to achieve a higher front density for a fixed ionizing laser energy. Operating close to the cutoff frequency of the waveguide such that the group velocity of the source radiation is only $0.5 c$ allows additional opportunities to test the theory. In free space with the source and ionizing radiation propagating collinearly, they will only interact with each other when they are counterpropagating. This is because the front will propagate at approximately the group velocity of the ionizing laser radiation in the plasma it has created, which must be less than $c$. However, when the group velocity of the source radiation is less than $c$, it can interact with the front even when they copropagate (the source radiation will be overtaken by the front). Theory predicts that for a given plasma density the degree of upshift in the copropagating geometry will be greater than in the counterpropagating case. In both cases the degree of upshift will be proportional to the plasma density in the front.

We refer to radiation which is initially propagating in the same direction as the front, and is thus overtaken, as a forward wave. Radiation which is initially counter-propagating with respect to the laser pulse is referred to as a backward wave (see Fig. 1). Theory predicts that the group velocity of a forward wave will always increase as it is upshifted by its interaction with the ionization front. Thus for large upshifts, the upshifted wave will follow along closely behind the front, leading to significant compression of the duration of the upshifted radiation. On the other hand, in the counterpropagating case the group velocity of the backward wave initially decreases as it is upshifted. This leads to an increase in its duration. Stated another way, the increase in the frequency of the forward wave is always greater than the increase in the cutoff frequency of the waveguide as it is filled with plasma. Thus the upshifted forward radiation is always farther from cutoff than the source radiation, and its group velocity is closer to $c$. Initially, it is just the opposite for the backward wave. In fact, as the plasma density in the front increases the group velocity of the backward wave approaches zero. Theory predicts that as the density of the front increases further, the backward wave begins to propagate in the same direction as the front. The group velocity of this turned-around backward wave increases with plasma density, and the wave is also significantly compressed in duration for large upshifts.

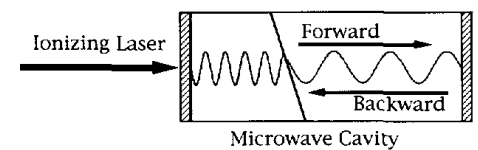

Fig. 1. Forward wave/backward wave convention.

In our experiments we have upshifted the $35 \mathrm{GHz}$ source radiation to more than $173 \mathrm{GHz}$. Using microwave interferometry to measure the spatially-averaged plasma density in the interaction region, we have confirmed that the upshift is proportional to front density, as predicted. We have measured the duration of the upshifted pulses with better than $1 \mathrm{nsec}$ resolution. The measured pulse lengths are in reasonable agreement with the theoretical predictions for both the forward and backward waves. We have observed the duration of the backward wave increasing with increasing plasma density, but have been unable to access the density regime where the backward wave should turn around and have a high group velocity in the forward direction. The observation of this "reflected" backward wave remains a critical test of the theory of underdense ionization fronts.

\section{THEORY}

The early theoretical work in this area concentrated on reflection from overdense ionization fronts [2], [3]. More recently, frequency upshifting by copropagating plasma waves and ionization fronts has been studied as well [8], [9]. In 1991 W. B. Mori showed theoretically that underdense ionization fronts can significantly increase the frequency and compress the duration of impinging electromagnetic radiation [4]. We extend his theoretical analysis to the case where the front and the source radiation interact inside a metallic waveguide. We derive the expected upshifted frequencies for the forward and backward waves in both the rest frame of the ionization front and in the laboratory frame using a space-time analysis of the upshifting process. We also derive the reflection and transmission coefficients for the waveguide case, including the coefficient for the static, spatially periodic magnetic field that is left behind in the plasma (the free streaming mode in the front's frame). Finally, we pay special attention to the proposed laboratory frame "reflection" of the transmitted backward wave into the direction of propagation of the front.

\section{A. Derivation of Upshifted Frequencies in Waveguide}

The derivation of the upshifted frequencies can be carried out either in the laboratory frame or in the rest frame of the ionization front. By making a Lorentz transformation to the front's rest frame, the derivation is simplified to that of a stationary boundary with neutral gas and a higher frequency electromagnetic wave impinging upon it from one side and plasma and the upshifted radiation streaming away on the other. However, in certain geometries the front may propagate faster than the speed of light, in which case Lorentz transformations to the front's frame are impossible. This does not contradict any physical principles; the front is simply the boundary at which the ionization takes place, and therefore 
carries no information or energy. Because one cannot Lorentz transform to a frame moving faster than $c$, the analysis for super-luminous fronts is simplest in the laboratory frame [3]. We use space-time plots for our laboratory frame analysis because they help one to visualize the upshifting and pulse compression over the full range of front velocities, from stationary to infinitely fast ("flash ionization" [15]-[17]).

Consider a long microwave cavity of unspecified cross section containing counterpropagating microwave radiation (a standing wave, for example). Such radiation consists of both a forward and a backward wave. An ionizing laser pulse passes along the axis of the cavity creating an underdense, relativistically propagating ionization front. Before the arrival of the laser pulse, the forward and backward radiation have the same frequency, $\omega_{f}=\omega_{b}=\omega$. The magnitude of their oppositely directed wave vectors is determined by the dispersion relation for electromagnetic radiation in the uniform waveguide, $\omega^{2}=\omega_{c}^{2}+c^{2} k_{f, b}^{2}$, where $\omega_{c}$ (Lorentz invariant along the waveguide axis) is the cutoff frequency for the particular operating mode. They both propagate at the group velocity, $v_{g}=c\left(1-\omega_{c}^{2} / \omega^{2}\right)^{1 / 2}$, which can be significantly less than $c$ for $\omega$ close to $\omega_{c}$. If we neglect depletion of the ionizing laser pulse, and assume that its frequency, $\omega_{l}$, is much higher than the cutoff frequencies of the waveguide modes $\left(\omega_{l} \gg \omega_{c}\right)$, then the front propagates at approximately the velocity of the ionizing laser pulse in the plasma. This velocity, given by

$$
v_{\text {front }} \simeq c\left\{1-\left(\omega_{c}^{2}+\omega_{p}^{2}\right) / \omega_{l}^{2}\right\}^{1 / 2}
$$

can be very close to $c$. Here we assume that the laser is primarily in the same mode as the microwave radiation.

The front's frame derivation proceeds by first making a Lorentz transformation to the rest frame of the ionization front. As mentioned earlier, in this frame the front is a stationary boundary with neutral gas streaming toward it on one side and plasma streaming away from it at $v_{\text {front }}$ on the other. When $v_{g}<v_{\text {front }}$ both the forward and the backward waves propagate toward the front with frequencies in this frame given by $\omega_{f}^{\prime}=\omega \gamma\left(1 \mp \beta v_{g} / c\right)$. Here $\gamma \equiv\left(1-\beta^{2}\right)^{-1 / 2}$ is the relativistic Lorentz factor for the front, and $\beta \equiv v_{\text {front }} / c$. If $\left(\omega_{p}^{2}+\omega_{c}^{2}\right)^{1 / 2}<\omega_{f, b}^{\prime}$, then the front is underdense and the radiation is transmitted into it. As with any stationary boundary, the transmitted radiation has the same frequency as the incident radiation, but its wave number adjusts to obey the dispersion relation for electromagnetic radiation in the streaming plasma, namely $\omega_{\text {plasma }_{f . b}}^{\prime 2}=\omega_{c}^{2}+\omega_{p}^{2}+$ $c^{2} k_{\text {plasma }_{f, b}}^{2}$. Thus, inside the plasma in the front's frame we have $\omega_{\text {plasma }_{f, b}}^{\prime}=\omega_{f, b}^{\prime}$ and $k_{\text {plasma }_{f, b}}^{\prime}=(1 / c)\left(\omega_{f, b}^{\prime 2}-\right.$ $\left.\omega_{c}^{2}-\omega_{p}^{2}\right)^{1 / 2}$. To evaluate the frequencies of the upshifted radiation in the plasma in the laboratory frame, we now Lorentz transform back and obtain

$$
\omega_{\text {plasma }_{f, b}}=\omega_{\text {plasma }_{f, b}}^{\prime} \gamma\left\{1-\beta\left(1-\frac{\omega_{c}^{2}+\omega_{p}^{2}}{w_{\text {plasma }_{f, b}}^{\prime 2}}\right)^{1 / 2}\right\} .
$$

Because all of the cavity and plasma boundaries are stationary in the laboratory frame, the frequency of the radiation will not change as it leaves the cavity. For underdense fronts $\left(\left(\omega_{p}^{2}+\omega_{c}^{2}\right)^{1 / 2} \ll \omega_{b}^{\prime}\right)$. (3) can be expanded to give

$$
\omega_{\text {plasma } f} \approx \frac{\omega}{2}\left(1 \mp \beta v_{g} / c\right)\left\{1+\frac{\omega_{c}^{2}+\omega_{p}^{2}}{\omega^{2}\left(1 \mp \beta v_{g} / c\right)^{2}}\right\} .
$$

Similarly, the upshifted wave numbers in the laboratory frame are

$$
k_{\text {plasma }_{b}} \approx \frac{\omega}{2 c}\left(1 \mp \beta v_{g} / c\right)\left\{1-\frac{\omega_{c}^{2}+\omega_{p}^{2}}{\omega^{2}\left(1 \mp \beta v_{g} / c\right)^{2}}\right\} .
$$

It follows from (4) that the upshifted frequencies increase linearly with plasma density and that $\omega_{\text {plasma }}$ is always greater than $\omega_{\text {plasma }_{b}}$. For highly relativistic fronts $(\beta \approx 1)$ the degree of upshift is relatively independent of $v_{\text {front }}$, depending only on the plasma density. The forward wave always propagates in the forward direction; $k_{\text {plasma }}$ is always negative. On the other hand, $k_{\text {plasma }_{b}}$ changes sign when the term in brackets in (5) changes sign. Therefore, as the front's density increases, the backward wave's group velocity decreases to zero then increases in the forward direction. This occurs when the group velocity of the backward wave in the front's frame is less than $v_{\text {front }}$ [4]. This type of "reflection" from an underdense front can give rise to large frequency upshifts and temporal compressions that are much different than those predicted by the usual Doppler effect. This topic will be discussed in more detail in a later section.

We now present a laboratory frame analysis using spacetime diagrams [11]. Fig. 2 is a space-time plot for an ionization front interacting with radiation confined within a microwave cavity operating near cutoff. The ionizing laser pulse, which passes through the cavity from right to left, is propagating at approximately $c$, and is represented by the thick line at $45^{\circ}$ to the space and time axes. The parallel vertical lines in the figure represent the stationary cavity windows from which a portion of the circulating microwave radiation reflects before the arrival of the laser pulse. The group velocity of the circulating radiation inside the cavity, $v_{g}$, is $0.5 c$. The parallel sets of lines between the cavity windows and below the laser pulse's space-time line in Fig. 2 show the propagation along the cavity axis of points of equal phase in the source radiation. Because the product of the group velocity and the phase velocity $v_{p}$ is $c^{2}$, the phase velocity of the source radiation, given by the inverse of the slope of the parallel lines, is greater than $c$.

As the source radiation is transmitted into the underdense front, its frequency is upshifted. The degree of upshift can easily be derived by taking a close look at the interaction of the radiation with the moving boundary. Fig. 3 shows a magnified detail of Fig. 2 along the front's space-time line. The parallel dashed lines track points of constant phase for the radiation inside the cavity that is propagating in the same direction as, and being overtaken by, the ionization front, the forward wave. The parallel dotted lines track points of constant phase for the upshifted, transmitted radiation. The derivation proceeds from three simple requirements: 1 ) the source radiation must obey 


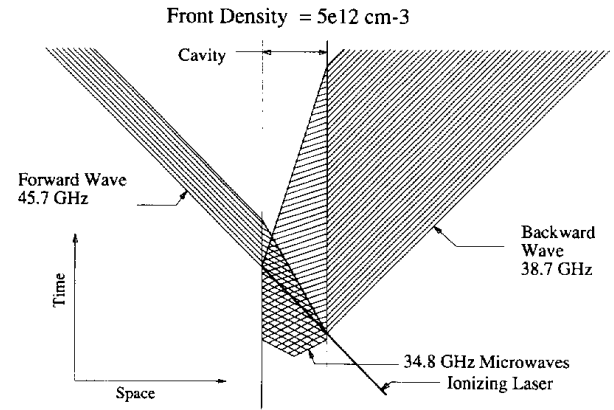

Fig. 2. Space-time diagram for an ionization front passing through a resonant microwave cavity.

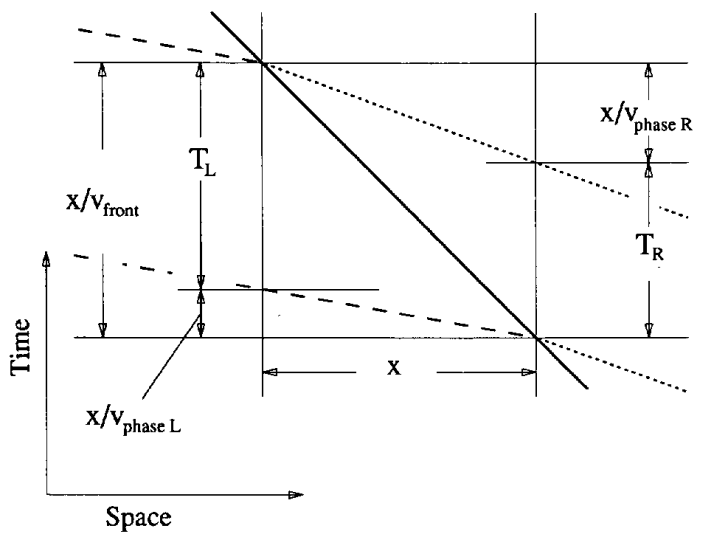

Fig. 3. Detail of Fig. 2 along the ionization front's space-time line for the forward radiation.

the dispersion relation (Lorentz-invariant in our geometry) for an electromagnetic wave in a conducting waveguide, $\omega^{2}=$ $\omega_{c}^{2}+c^{2} k^{2}$, where $\omega_{c}$ is the cutoff frequency for the particular operating mode in the waveguide; 2 ) the number of cycles of the source radiation impinging on the front must equal the number of cycles of upshifted radiation leaving the front; and 3) the upshifted radiation must obey the dispersion relation for an electromagnetic wave in a plasma filled waveguide (also Lorentz-Invariant), $\omega_{u p}^{2}=\omega_{c}^{2}+\omega_{p}^{2}+c^{2} k^{2}$, where $\omega_{p}$ is the plasma frequency. The first and third requirements thus dictate the slope of the parallel lines between the cavity windows below and above the front's space-time line in Fig. 3, and the second requirement is simply that the spacing between the lines, measured along the front's space-time line, must be equal to both sides.

The full range of front velocities can be considered by simply changing the slope of the front's space-time line. The two limiting cases are a stationary boundary and one that propagates at an infinitely high velocity. For the stationary boundary, the front's space-time line is vertical and requirement 2 is simply that the frequency is fixed. As is the usual case for stationary interfaces, the wave number must adjust itself in order to obey requirement 3 . For the infinitely fast front, also known as "flash ionization" [15], the boundary is horizontal in the space-time plot. Thus, the wave number is fixed by requirement 2 and the frequency adjusts in order to satisfy requirement 3 .

It can easily be seen in Fig. 3 that the temporal periods of the radiation on the left and right sides of the front are given by

$$
T_{L}=\frac{2 \pi}{\omega_{L}}=X\left(\frac{1}{v_{\text {front }}}-\frac{1}{v_{\text {phase }_{L}}}\right)
$$

and

$$
T_{R}=\frac{2 \pi}{\omega_{R}}=X\left(\frac{1}{v_{\text {front }}}-\frac{1}{v_{\text {phase }_{R}}}\right) .
$$

Using these expressions, we can write the upshifted frequency as a function of the source frequency as

$$
\omega_{R}=\omega_{L} \frac{\left(1 / v_{\text {front }}-1 / v_{\text {phase }_{L}}\right)}{\left(1 / v_{\text {front }}-1 / v_{\text {phase }_{R}}\right)} .
$$

Viewed another way, this relationship follows from the requirement that the phase be continuous across the boundary, which is a consequence of the requirement that $\mathrm{E}$ and $\mathrm{B}$ be continuous at the front. The dispersion relations to the left of (before) and to the right of (after) the front are

$$
\omega_{L}^{2}=\omega_{c}^{2}+c^{2} k_{L}^{2}
$$

and

$$
\omega_{R}^{2}=\omega_{c}^{2}+\omega_{p}^{2}+c^{2} k_{R}^{2}
$$

By eliminating $k_{R}$ and $k_{L}$ from the above expressions, we obtain a quadratic equation in $\omega_{R}$ which can be solved to give

$$
\begin{aligned}
\omega_{R}= & \omega_{L} \gamma^{2}\left(1-\beta v_{g} / c\right) \\
& \cdot\left\{1-\beta\left[1-\frac{\omega_{c}^{2}+\omega_{p}^{2}}{\omega_{L}^{2} \gamma^{2}\left(1+\beta v_{g} / c\right)^{2}}\right]^{\frac{1}{2}}\right\} .
\end{aligned}
$$

Here $\beta=v_{\text {front }} / c$ and $\gamma=\left(1-\beta^{2}\right)^{-1 / 2}$ is the relativistic Lorentz factor associated with the front. $v_{g}$ is the group velocity of the source radiation in the cavity, and is related to $v_{\text {phase }_{L}}$ by $v_{g} v_{\text {phase }_{L}}=c^{2}$. A similar derivation for the radiation inside the cavity which is propagating in the opposite direction to the ionization front (left to right in Fig. 2), the backward wave, yields an upshifted frequency given by

$$
\begin{aligned}
\omega_{R}= & \omega_{L} \gamma^{2}\left(1+\beta v_{g} / c\right) \\
& \cdot\left\{1-\beta\left[1-\frac{\omega_{c}^{2}+\omega_{p}^{2}}{\omega_{L}^{2} \gamma^{2}\left(1+\beta v_{g} / c\right)^{2}}\right]^{\frac{1}{2}}\right\} .
\end{aligned}
$$

The upshifted forward wave is shown by the parallel lines above and to the left of the front's line in Fig. 2, and the backward wave is shown by the parallel lines above and to the right. These lines represent the propagation of points of equal phase, and are bounded by lines which represent the group velocity of the radiation. Equations (11) and (12) both predict upshifted frequencies that are proportional to the front density, with the forward wave always upshifted to a higher 

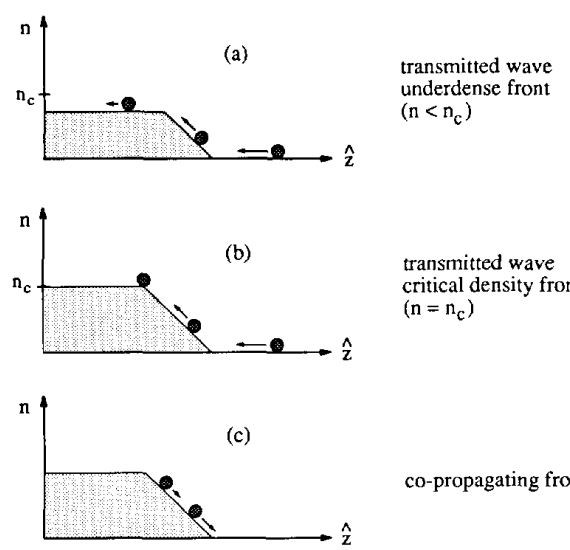

co-propagating front

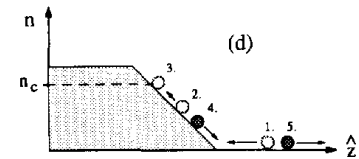

Fig. 4. Radiation impinging upon an ionization front of finite spatial extent (viewed in the rest frame of the front): (a) a wave transmitted into an underdense front, (b) a wave transmitted into a front of critical density (the wave has zero group velocity after passing through the front), (c) a wave propagating down the density gradient of the front (copropagating scenario), (d) a wave reflected by an overdense front.

frequency than that of the backward wave for a given plasma density. We note that $\beta$ is not limited to being less than unity, in fact, we recover the flash-ionization results for $\beta \rightarrow \infty$ [15]. Equations (11) and (12) give exactly the same result as (3), as expected.

To derive the maximum possible upshift for both underdense and overdense ionization fronts, consider a wave impinging upon a front of finite spatial extent. Viewed from the front's frame, it is simply a wave propagating toward a stationary density gradient. This is illustrated in Fig. 4 where the interaction is represented in the front's frame by a ball (wave) rolling toward a hill (ionization front). Following our previous convention, the incident wave frequency in this frame is $\omega_{f}^{\prime}=\omega \gamma\left(1 \mp \beta v_{g} / c\right)$, as before. For an underdense front, the velocity of the wave (ball) decreases as it propagates into the front (up the hill) as shown in Fig. 4a. For higher density fronts, the wave will propagate into the density gradient until it reaches the point where the plasma density is equal to the critical density, $n_{c}$; that is, when $\omega_{f}^{\prime}=\left(\omega_{c}^{2}+\omega_{p}^{2}\right)^{1 / 2}$ (see Fig. 4(b)). This density separates the underdense and overdense regimes. At this density the group velocity of the radiation in this frame goes to zero.

Therefore, the maximum upshift (seen in the laboratory frame) for an underdense ionization front will occur when $\omega_{f}^{\prime}=\left(\omega_{c}^{2}+\omega_{p}^{2}\right)^{1 / 2}$. We obtain this maximum upshifted frequency by substituting $\left(\omega_{c}^{2}+\omega_{p}^{2}\right)^{1 / 2}$ for $\omega_{f}^{\prime}$ in (3). Thus

$$
\omega_{f}(\max )=\left(\omega_{c}^{2}+\omega_{p}^{2}\right)^{1 / 2} \gamma\left\{1-\beta\left(1-\frac{\omega_{c}^{2}+\omega_{p}^{2}}{\omega_{c}^{2}+\omega_{p}^{2}}\right)^{1 / 2}\right\}
$$

or

$$
\begin{aligned}
\omega_{f}(\max )=\gamma\left(\omega_{c}^{2}+\omega_{p}^{2}\right)^{1 / 2} & =\left[\frac{\omega_{c}^{2}+\omega_{p}^{2}}{1-\beta^{2}}\right]^{1 / 2} \\
& =\left[\frac{\omega_{c}^{2}+\omega_{p}^{2}}{1-\left(v_{\text {front }} / c\right)^{2}}\right]^{1 / 2} .
\end{aligned}
$$

Substituting $v_{\text {front }}$ given by (2) we see that

$$
\omega_{f}(\max )=\omega_{l} \text {. }
$$

Thus the maximum upshifted frequency obtainable using underdense fronts is the frequency of the ionizing radiation [4]. At that frequency the upshifted radiation is propagating at the same velocity and in the same direction as the front (in the laboratory frame).

For densities above the critical density, the wave vector changes sign (the ball begins to roll back down the hill). This situation, where the wave is propagating through the density gradient in the same direction as the front (when viewed from the front's frame) is referred to as the co-propagating front scenario (see Fig. 4(c)). Although the frequency of the radiation is unchanged in this frame, the wave number is changing to continue to obey the dispersion relation which is a function of the plasma density. When we make a Lorentz transformation back to the laboratory frame, this change in the wave number will manifest itself in a change in both the frequency and the wave number of the upshifted radiation [7], [8], [18]

If the interaction region is long enough, the reflected radiation will propagate completely down the density gradient (to the bottom of the hill). Once the wave has passed all the way back through the gradient, it is again in free space and is a truly reflected wave (see Fig. 4(d)). The upshift of such a reflected wave is a result of the relativistic Doppler effect [3]. This can be derived by Lorentz transforming to the front's frame, reversing the direction of the transformed wave vector, then Lorentz transforming back to the laboratory frame. For the waveguide case, this upshift is given by

$$
\omega_{f}(\text { refl })=\omega \frac{\left(1 \mp 2 \beta v_{g} / c+\beta^{2}\right)}{\left(1-\beta^{2}\right)}
$$

If we again assume that this reflection takes place at the density where

$$
\omega_{f}^{\prime}=\omega \gamma\left(1 \mp \beta v_{g} / c\right)=\left(\omega_{c}^{2}+\omega_{p}^{2}\right)^{1 / 2}
$$

then $\gamma\left(\omega_{c}^{2}+\omega_{p}^{2}\right)^{1 / 2}=\omega_{l}$, and we can write

$$
\omega_{f}(\mathrm{refl})=\omega_{l} \frac{\left(1 \mp 2 \beta v_{g} / c+\beta^{2}\right)}{\left(1 \mp \beta v_{g} / c\right)} .
$$

For relativistic fronts, where $\beta \approx 1$, this reduces to

$$
\omega_{f}(\text { refl })=2 \omega_{l} \frac{\left(1 \mp v_{g} / c\right)}{\left(1 \mp v_{g} c\right)}=2 \omega_{l}
$$

The upshifted frequency is plotted versus the plasma frequency of the front for three values of incident radiation 


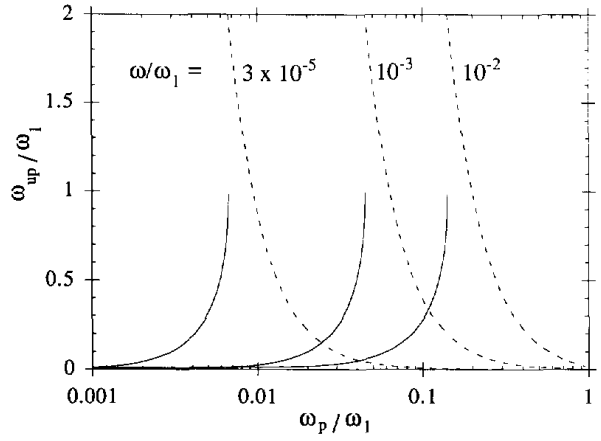

Fig. 5. Upshifted frequency vs. ionization front plasma frequency for three incident radiation frequencies. Frequencies are normalized to the ionizing laser frequency. The incident frequency for our experiment is $\omega / \omega_{l}=3 \times 10^{-5}$. The solid lines represent the underdense upshifts and the dashed lines represent the overdense (Doppler reflection) upshifts.

frequency in Fig. 5. For our experimental parameters, $\omega / \omega_{l}=$ $3 \times 10^{-5}$. The solid curves which terminate at $\omega_{l}$ are the underdense upshifts given by (3). The dashed curves which begin at $2 \omega_{l}[4,14]$ are the reflected wave upshifts given by (14). They decrease at higher densities because they are strongly dependent on the front's velocity (see (2)). For the reflected upshifts we have assumed that the interaction length is sufficient to allow the wave to travel all the way back through the density gradient. If the wave only traverses part of it, the upshift will be between $\omega_{l}$ and $2 \omega_{l}$.

\section{B. Derivation of Reflection and Transmission Coefficients in Wave-guide}

The reflection and transmission coefficients for a TE wave polarized perpendicular to the plane of incidence ( $s$ polarization) can easily be calculated in the front's frame [11] (recall that in this frame the problem is reduced to that of a stationary boundary). We first derive the modes that can exist in the streaming plasma. The momentum equation for the electrons in the front's frame is

$$
\frac{\partial \vec{v}}{\partial t}+\vec{U} \frac{\partial \vec{v}}{\partial z}=-\frac{e}{m_{0} \gamma}\left(\vec{E}+\frac{1}{c} \vec{U} \times \vec{B}\right) .
$$

$\vec{U}$ is the velocity of the neutral gas, which in this frame is equal to the velocity of the front, and $m_{0}$ is the rest mass of the electrons. We use two of Maxwell's equations,

$$
\nabla \times \vec{E}=-\frac{1}{c} \frac{\partial \vec{B}}{\partial t}
$$

and

$$
\nabla \times \vec{B}=\frac{1}{c} \frac{\partial \vec{E}}{\partial t}-\frac{4 \pi}{c} \vec{J}
$$

where $\vec{J}$, the free current, is ne $\vec{v}$. By taking the curl of (18), considering only TE waves $(\nabla \cdot \vec{E}=0)$, and substituting the right side of (19) for $\nabla \times \vec{B}$, we can write

$$
\nabla^{2} \vec{E}=\frac{1}{c^{2}} \frac{\partial^{2} E}{\partial t^{2}}-\frac{4 \pi n e}{c^{2}} \frac{\partial v}{\partial t} .
$$

We Fourier analyze by assuming solutions of the form $e^{i(\vec{k} \cdot \vec{r}-\omega t)}$. Differentiating (20) gives

$$
\left(\frac{\omega^{2}}{c^{2}}-k^{2}\right) \vec{E}=\frac{-i 4 \pi n e \omega}{c^{2}} \vec{v} .
$$

We can now differentiate (17) and eliminate $\vec{E}$ and $\vec{B}$ using (18) and (21) to write, after a bit of algebra,

$$
\left(\omega-U k_{\|}\right)\left(1-\frac{\omega_{p}^{2}}{\omega^{2}-c^{2} k^{2}}\right)=0
$$

where the subscript $\|$ denotes along the direction of $U$, which is opposite to the direction of propagation of the front. This equation has three roots,

$$
\begin{gathered}
k_{1 \|}=\frac{\omega}{U} \\
k_{2}=\frac{\omega}{c}\left(1-\frac{\omega_{p}^{2}}{\omega^{2}}\right)^{\frac{1}{2}}
\end{gathered}
$$

and

$$
k_{3}=-\frac{\omega}{c}\left(1-\frac{\omega_{p}^{2}}{\omega^{2}}\right)^{\frac{1}{2}}
$$

We neglect the third root because we assume a positive value for the wave number of the source wave.

Now that we know that there are two possible modes in the streaming plasma, we can apply the boundary conditions to solve for the reflection and transmission coefficients. We assume a discontinuous boundary between the neutral gas and the plasma, which lies in the $x-y$ plane. $U$ is along the $z$ axis and an electromagnetic wave with its electric field along the $x$ axis is incident upon the boundary with its wave vector in the $y-z$ plane (s-polarization). Three independent conditions that must be satisfied at the plasma-neutral boundary are: 1) continuity of the tangential component of $\vec{E}, 2$ ) continuity of the normal component of $\vec{B}$, and 3 ) the total current must be zero because the electrons have negligible initial velocities with respect to the ions when they are created at the boundary. Considering an incident mode (subscript $i$ ), a reflected mode (subscript $r$ ), and the two transmitted modes derived earlier (subscripts 1 and 2) these boundary conditions can be written as:

$$
\begin{aligned}
& \left(\vec{E}_{i}+\vec{E}_{r}\right)_{\perp}=\left(\vec{E}_{1}+\vec{E}_{2}\right)_{\perp} \\
& \left(\vec{B}_{i}+\vec{B}_{r}\right)_{\|}=\left(\vec{B}_{1}+\vec{B}_{2}\right)_{\|}
\end{aligned}
$$

and

$$
\nabla \times\left(\vec{B}_{1}+\vec{B}_{2}\right)=\frac{1}{c} \frac{\partial}{\partial t}\left(\vec{E}_{1}+\vec{E}_{2}\right) .
$$

In our geometry, (26) and (27) become

$$
E_{i}+E_{r}=E_{1}+E_{2}
$$

and

$$
B_{i} \frac{k_{i \|}}{k_{i}}-B_{r} \frac{k_{r \|}}{k_{r}}=B_{1} \frac{k_{1 \|}}{k_{1}}-B_{2} \frac{k_{2 \|}}{k_{2}} .
$$


Realizing that $B=(c k / \omega) E$ for each mode and that $k_{i \|}=$ $k_{r \|}$, we can eliminate the reflected mode from the two equations above and write

$$
E_{1}=\frac{2 E_{i}-E_{2}\left(1+\frac{k_{2 \|}}{k_{i \|}}\right)}{\left(1+\frac{k_{1 \|}}{k_{i \|}}\right)}
$$

Equation 28 can be differentiated and rearranged to give

$$
E_{1}=E_{2}\left(\frac{\omega^{2}-c^{2} k_{2}^{2}}{c^{2} k_{1}^{2}-\omega^{2}}\right)
$$

We can now equate (31) and (32) and reduce them to

$$
\frac{E_{2}}{E_{i}}=\frac{2}{\left(1+\frac{k_{2 \|}}{k_{i \|}}\right)+\left(1+\frac{k_{1 \|}}{k_{i \|}}\right)\left(\frac{\omega^{2}-c^{2} k_{2}^{2}}{c^{2} k_{1}^{2}-\omega^{2}}\right)} .
$$

Using this and (32), we obtain

$$
\frac{E_{1}}{E_{i}}=\frac{2}{\left(1+\frac{k_{1 \|}}{k_{i \|}}\right)+\left(1+\frac{k_{2} \|}{k_{i \|}}\right)\left(\frac{c^{2} k_{1}^{2}-\omega^{2}}{\omega^{2}-c^{2} k_{2}^{2}}\right)} .
$$

And finally, (29) simply gives us

$$
\frac{E_{r}}{E_{i}}=\frac{E_{1}}{E_{i}}+\frac{E_{2}}{E_{i}}-1
$$

Thus (33)-(35) give us the reflection and transmission coefficients in the front's frame. In order to obtain the laboratory frame coefficients, we must transform the fields back to the laboratory frame.

We make the Lorentz transformation using the relations

$$
\vec{E}_{\mathrm{lab}}=\gamma(\vec{E}+\vec{\beta} \times \vec{B})-\frac{\gamma^{2}}{\gamma+1} \vec{\beta}(\vec{\beta} \cdot \vec{E})
$$

and

$$
\vec{B}_{\text {lab }}=\gamma(\vec{B}+\vec{\beta} \times \vec{E})-\frac{\gamma^{2}}{\gamma+1} \vec{\beta}(\vec{\beta} \cdot \vec{B}) .
$$

Using these relations, we can write the electric fields in the laboratory frame as

$$
\begin{gathered}
\vec{E}_{\text {ilab }}=\vec{E}_{i} \gamma\left(1-\beta \frac{k_{i \|} c}{\omega}\right) \\
\vec{E}_{\text {rlab }}=\vec{E}_{r} \gamma\left(1+\beta \frac{k_{i \| c}}{\omega}\right) \\
\vec{E}_{1 \mathrm{lab}}=0
\end{gathered}
$$

and

$$
\vec{E}_{2 \text { lab }}=\vec{E}_{2 \gamma}\left(1-\beta \frac{k_{2 \|} c}{\omega}\right)
$$

Because $\vec{E}_{1}$ transforms to zero in the laboratory frame, we see that this mode is simply a static magnetic field. We can use the relation $B_{1}=\left(c k_{1} / \omega\right) E_{1}$ to obtain $B_{1}$, then transform back to the laboratory frame to give

$$
\vec{B}_{1 \mathrm{lab}}=E_{1} \gamma\left\{\left(\frac{c k_{1 \|}}{\omega}-\beta\right) \hat{\perp}+\frac{c k_{1 \|}}{\gamma \omega} \hat{\|}\right\}
$$

or

$$
B_{1 \mathrm{ab}}=E_{1} \gamma\left\{\left(\frac{c k_{1 \|}}{\omega}-\beta\right)^{2}+\left(\frac{c k_{1 \perp}}{\gamma \omega}\right)^{2}\right\}^{\frac{1}{2}} .
$$

We can now write, after a considerable amount of algebraic manipulation, the laboratory frame reflection and transmission coefficients as [11]

$$
\begin{gathered}
r \equiv \frac{E_{r \mathrm{lab}}}{E_{\text {ilab }}}=\frac{\left(\sqrt{\epsilon_{i}^{\prime}}-\sqrt{\epsilon_{t}^{\prime}}\right)}{\left(\sqrt{\epsilon_{i}^{\prime}}+\sqrt{\epsilon_{t}^{\prime}}\right)} \\
t_{2} \equiv \frac{E_{2 \mathrm{lab}}}{E_{\text {ilab }}}=\frac{2 \sqrt{\epsilon_{i}^{\prime}}}{\left(\sqrt{\epsilon_{i}^{\prime}}+\sqrt{\epsilon_{t}^{\prime}}\right)}
\end{gathered}
$$

and

$$
t_{1} \equiv \frac{B_{1 \mathrm{lab}}}{E_{i \mathrm{lab}}}=\left\{\frac{1-\beta^{2}}{1-\beta^{2} \epsilon_{i}^{\prime}}\right\}^{\frac{1}{2}} 2 \beta \sqrt{\epsilon_{i}^{\prime}} \frac{\left(\sqrt{\epsilon_{i}^{\prime}}-\sqrt{\epsilon_{t}^{\prime}}\right)}{1-\beta \sqrt{\epsilon_{t}^{\prime}}}
$$

Here $\epsilon_{i}^{\prime} \equiv 1-\omega_{c}^{2} / \omega^{\prime 2}$ and $\epsilon_{t}^{\prime} \equiv 1-\left(\omega_{c}^{2}+\omega_{p}^{2}\right) / \omega^{2}$ with primed quantities specified in the front's frame.

For the range of plasma densities and $\sqrt{\epsilon_{i}^{\prime}}$ values used in our experiments, $r \approx 0$ and $t \approx 1$. Note that (44)-(46) reduce to the free space expressions in the limit $\epsilon_{i} \rightarrow 1\left(\omega_{c}=0\right)$. Although the ratio of the transmitted to incident powers at the front is always expected to be nearly unity, for large upshifts most of the incident energy is left in the free-streaming modes's static $B$ field as the transmitted wave's duration shortens. The above model assumes that the ionization front is discontinuous. Similar results are obtained for finite length underdense fronts [4], except that the energy in the freestreaming mode is instead converted to thermal energy in the plasma, as is the case for finite length overdense fronts [3]

\section{Derivation of Upshifted Pulselengths in Waveguide}

In deriving the expected duration of the upshifted radiation for a given interaction geometry, we first consider the group velocity of the upshifted radiation within the waveguide. The group velocity of the upshifted radiation in the plasma in the front's frame is given by

$$
v_{g f, b}^{\prime}=\frac{d \omega_{f, b}^{\prime}}{d k_{f, b}^{\prime}}=c^{2} \frac{k_{f, b}^{\prime}}{\omega_{f, b}^{\prime}} .
$$

By transforming this velocity back to the laboratory frame, we obtain the group velocity of the upshifted radiation inside the plasma-filled waveguide in the laboratory frame,

$$
v_{g f, b}=\frac{v_{g f, b}^{\prime}+v_{\text {front }}}{1+v_{g f, b}^{\prime} v_{\text {front }} / c^{2}} .
$$

Equation 48 is plotted in Fig. 6(a) as a function of front density for typical experimental parameters. The group velocity of the forward wave, which is always greater than that of the source radiation, increases as the plasma density in the front increases. On the other hand, the group velocity of the backward wave initially decreases as the front density increases. It goes to zero when the front density is such that

$$
\omega_{p}^{2}=\omega^{2}\left(1+\beta v_{g} / c\right)^{2}-\omega_{c}^{2} .
$$

At this point, the group velocity of the backward wave changes 

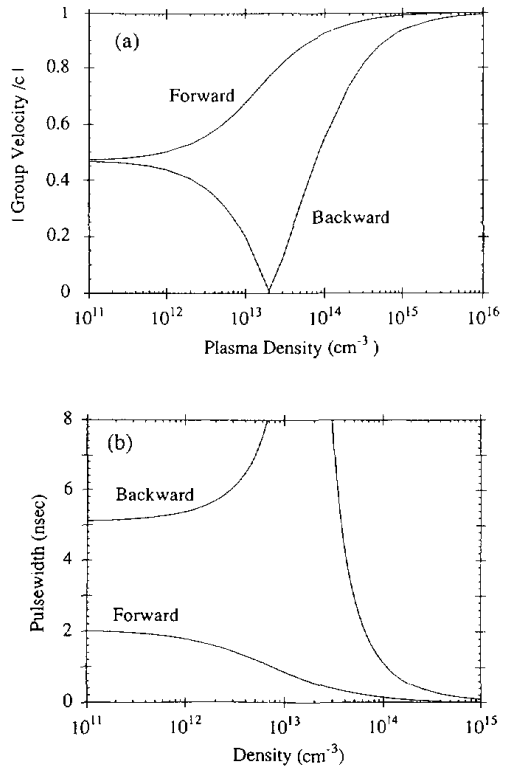

Fig. 6. (a) Group velocity of the forward and backward upshifted radiation vs. ionization front plasma density, (b) expected upshifted radiation pulsewidths vs. front density for our experimental parameters.

sign, then increases in the forward direction as the plasma density continues to increase.

Once we know the group velocity of the upshifted radiation, we can easily determine the duration of the upshifted pulses. First consider the overtaken forward wave. For underdense fronts, the highest group velocity is that of the ionizing radiation. If we assume negligible depletion of the ionizing pulse or very short ionizing pulses, then the front will propagate at this same velocity. Thus the upshifted forward wave will begin to exit the waveguide at the instant the ionizing laser arrives at the laser output window (the forward window of the waveguide). The trailing edge of the forward upshifted pulse, which was generated at the instant the laser pulse entered the waveguide through the backward window and then propagated a distance $x=v_{g f} L / v_{\text {front }}$ before the forward wave began to leave the guide, will exit the waveguide after traveling the remaining $L-x$ at $v_{g f} . L$ is the length of the waveguide. Similarly, for the backward wave, the pulse will begin to leave the waveguide at the instant the laser arrives at the backward window. The trailing edge of the backward pulse will leave the forward window, traveling toward the backward window, at the instant the laser pulse reaches the forward window. If the plasma density is high enough to turn the backward wave around, then the determination of its pulse length would follow the argument for the forward wave, but replacing $v_{g f}$ with $v_{g b}$. Thus a general expression for the upshifted pulse lengths is

$$
\tau_{\text {pulse }_{f, b}}=L\left|\frac{1}{v_{g f, b}}-\frac{1}{v_{\text {front }}}\right| .
$$

Equation 50 is plotted for typical experimental parameters ( $L=46 \mathrm{~cm}$, source $v_{g}=0.43 c$ ) in Fig. 6(b). The forward upshifted pulsewidth is $\sim 2$ ns for low plasma density and decreases dramatically at higher front densities. The pulsewidth of the upshifted backward wave starts at $\sim 5 \mathrm{~ns}$ and initially increases, becoming infinitely long as the wave approaches cutoff. At higher densities where the backward wave has turned around and attained a reasonably large group velocity in the forward direction, the pulsewidth is also significantly compressed.

\section{D. "Reflection" of the Backward Wave}

One of the most striking predictions of the underdense ionization front theory is the "reflection" of the backward wave. It is at first difficult to imagine that a wave propagating towards and transmitted through an approaching dielectric discontinuity will suddenly find itself propagating in the opposite direction. Consider a slab of plasma, or any other dielectric material, that is propagating through free space at a velocity, $v_{\text {slab }}$, toward an impinging electromagnetic wave [19]. Assume that the radiation is transmitted into the slab, as is the case for underdense ionization fronts. If the group velocity of the transmitted radiation inside the dielectric is greater than $v_{\text {slab }}$, then the radiation, which will have been upshifted in frequency at the moving boundary, will continue to propagate opposite to $v_{\text {slab }}$ (at a slower velocity). When the upshifted radiation is transmitted through the other side of the slab (another moving boundary), its frequency and group velocity will return to their original values. Now consider what an observer would see if the group velocity of the upshifted radiation were less than $v_{\text {slab}}$. Although the radiation is transmitted into and propagates through the slab, the observer in the laboratory frame would see it traveling in the same direction (at a slower velocity) as the slab while it is inside. Because it has a slower velocity than the slab, it will eventually be overtaken and once it leaves the slab it would return to its original characteristics, as in the previous example.

Our ionization front experiments differ from the moving plasma slab examples. The plasma itself is stationary, rather than moving at $v_{\text {slab }}$, and only the ionization boundary propagates at $v_{\text {front }}$. However, this has no effect on the upshifted frequencies or group velocities because the plasma frequency is Lorentz-invariant. Yet, because the recombination time of the plasma is long compared with the cavity transit times and the waveguide windows are stationary in the laboratory frame, the backward wave can turn around in the laboratory frame. When the upshifted group velocity is less than the front velocity, the observer will see the upshifted wave traveling through the plasma in the same direction as the front, as for the slab example. In the slab example the radiation then encountered the back edge of the slab and the group velocity returned to $c$ in the backward direction. But in the ionization front case, the plasma recombination time is long and the second boundary encountered is the stationary cavity window (or plasma/neutral interface). Because the radiation was propagating toward this window while inside the plasma, it is transmitted through the window and propagates at $c$ in the forward direction. The upshifted frequency and pulsewidth are very different from what would result from a true reflection from an overdense front or a moving mirror.

The space-time diagrams introduced earlier are also useful for visualizing the backward wave turn-around. Fig. 7(a) is a 


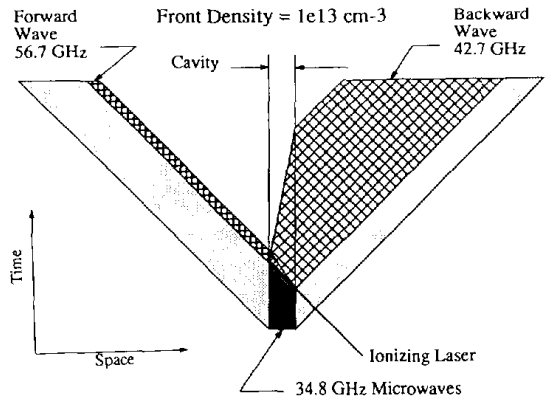

(a)

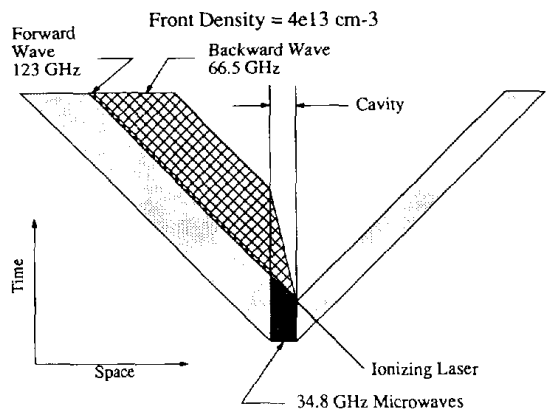

(b)

Fig. 7. Space-time diagrams (a) for ionization front density of $1 \times$ $10^{13} \mathrm{~cm}^{-3}$, (b) for front density of $4 \times 10^{13} \mathrm{~cm}^{-3}$. Note that at this higher density the upshifted backward wave is "reflected" into the forward direction.

space-time plot for a front density of $1 \times 10^{13} \mathrm{~cm}^{-3}$, where the group velocity of the backward wave is small, but it is still propagating in the backward direction. The stippled area indicates source radiation that leaks out of the cavity before the arrival of the laser pulse. For a front density of $4 \times 10^{13} \mathrm{~cm}^{-3}$, shown in Fig. 7(b), the backward wave has turned around and is propagating in the same direction as the ionizing laser. At this density the forward wave has already been significantly compressed in duration. Also, because the backward wave is now following along behind the front, its duration decreases significantly as its group velocity approaches that of the front at higher plasma densities.

\section{EXPERIMENT}

Our experimental investigation of the interaction of underdense, relativistically propagating ionization fronts has focused on two similar but distinct experimental configurations. Both utilize an ultraviolet laser pulse that ionizes a column of neutral gas enclosed by a waveguide. In both cases we investigate the interaction of $35 \mathrm{GHz}$ microwave radiation which interacts with the front inside the waveguide.

The configuration that was used the first experiments utilizes a resonant cylindrical waveguide cavity which is fed through the side wall [12], [13]. Because the cavity contains a standing microwave field, the front always interacts with a forward and a backward wave simultaneously.

The second configuration utilizes a waveguide of rectangular cross-section which is fed along the axis via a directional coupler [20]. In this case we are able to launch the microwave radiation in only either the forward or the backward direction.

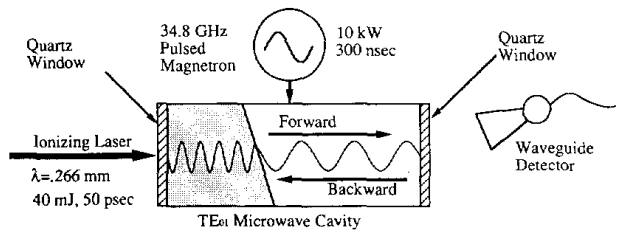

Fig. 8. Schematic of the experimental arrangement utilizing a resonant microwave cavity.

This allows us to better isolate the contributions of the two waves to our detected signals.

\section{A. Resonant Microwave Cavity Setup}

The experimental arrangement utilizing a resonant microwave cavity is shown schematically in Fig. 8 . The cavity consists of a 1.2-cm-diameter, $35-\mathrm{cm}$-long copper cylinder that is closed by a $0.3-\mathrm{cm}$-thick quartz window at each end. Microwaves from a pulsed magnetron are fed into the cavity through the side wall at the mid-plane and excite the $T E_{01}$ mode. The $T E_{01}$ mode, sometimes referred to as the low-loss mode, has an azimuthal electric field inside the waveguide [21]. It was chosen because it is low-loss and it does not require a current to flow between the end plate (a quartz window in this case) and the cylindrical cavity wall in order to maintain a high $Q$. The measured $Q$ of the cavity is $\sim 1000$. The magnetron gives a flat-top pulse with an instantaneous power of $\sim 10 \mathrm{~kW}$ and a duration of $\sim 300 \mathrm{~ns}$. The cavity is heated to $100^{\circ} \mathrm{C}$ in order to support azulene (which is solid at room temperature) vapor at pressures from a few to several hundred mT. Azulene is used because it is easily ionized by $.266 \mu \mathrm{m}$ radiation.

The ionizing laser pulse is derived from a mode-locked neodymium:yttrium aluminum garnet (Nd:YAG) laser giving a train of 100 psec-long pulses at a wavelength of $1.06 \mu \mathrm{m}$. Single pulses are selected from the train and amplified to the $200 \mathrm{~mJ}$ level in a Nd:YAG regenerative amplifier. These high-energy pulses are then frequency doubled twice using potassium titanium phosphate (KTP) crystals to give 50psec-long (FWHM) pulses containing $40 \mathrm{~mJ}$ of energy at a wavelength of $.266 \mu \mathrm{m}$ in the ultraviolet. The output beam diameter is $\sim 1 \mathrm{~cm}$ and thus fills the cavity aperture. The laser system is capable of producing these amplified pulses at a repetition rate of $10 \mathrm{~Hz}$. During experiments, the laser is typically fired once every 15 to $30 \mathrm{~s}$ in order to allow products of the ionization process to be pumped out of the waveguide.

Upshifted radiation is monitored by a series of diode detectors, each preceded by a length of rectangular waveguide and a horn, which are mounted at various positions at both ends of the cavity. To obtain subnanosecond resolution we use high-bandwidth SMA coaxial cables to transmit the signals to $1 \mathrm{GHz}$-bandwidth analog oscilloscopes (Tektronix 7104) which use a micro-channel plate to intensify the display. Terminating the signals into $50 \Omega$ significantly reduces the sensitivity of the detectors, but gives high temporal resolution. The signals typically require attenuation of 30 to $40 \mathrm{~dB}$ in order to stay in the linear regime of the detector response. This is accomplished using a precision resistive-wafer-style variable attenuator mounted between the detector and the cutoff wave- 

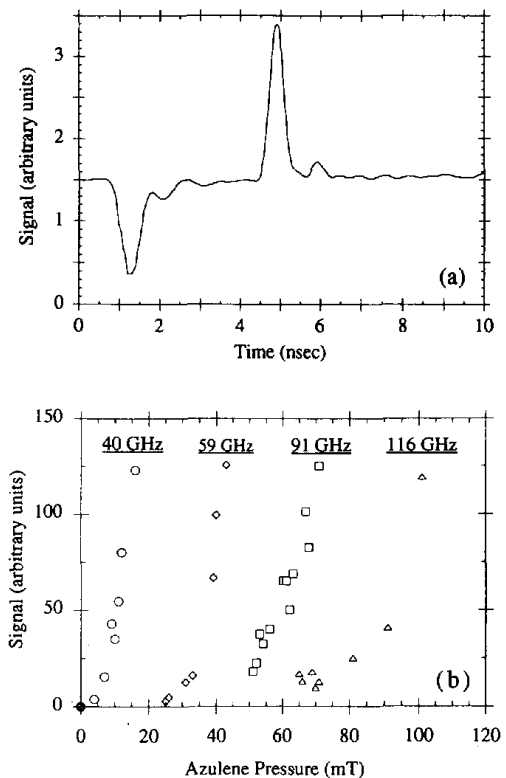

Fig. 9. (a) Typical upshifted signal using a $173 \mathrm{GHz}$ cutoff waveguide. The negative peak is a laser fiducial. (b) Plot of the onset of upshifted signals on the $40 \mathrm{GHz}, 59 \mathrm{GHz}, 91 \mathrm{GHz}$, and $116 \mathrm{GHz}$ detector channels vs. azulene pressure.

guide. The signals are recorded using digitizing CCD cameras mounted directly to the oscilloscope display. The digital images, captured via frame-grabber boards in a computer are then processed to extract the transient wave forms. These are then transported to an Apple Macintosh computer where they are scaled and further processed using National Instruments' LabView software. The bandwidth-limited temporal resolution of our detection system is $\sim 400$ psec (FWHM).

A typical experimental run begins with the heated cavity evacuated to a base pressure of a few mT. Azulene vapor is then slowly introduced into the cavity through a precision leak valve. When the azulene pressure is low, the upshifted frequency of both the forward and backward waves is below the cutoff frequency of the detector waveguide and thus is not detected. Once the plasma inside the cavity is sufficiently dense to shift the wave above the detector cutoff, the signa rises sharply then decreases as the response of the detector rolls off with the higher frequencies that are generated at higher pressures. A typical upshifted wave form recorded on the $173 \mathrm{GHz}$ detector channel is shown in Fig. 9(a). The early negative-going peak is a laser fiducial which triggers the oscilloscopes in synchronization with the ionizing laser pulse. For large upshifts (high azulene pressures) the upshifted signal is typically a single sub-nanosecond pulse, as shown.

In Fig. 9(b) we plot the onset of the upshifted signals measured in the forward direction for four detector channels at $40 \mathrm{GHz}, 59 \mathrm{GHz}, 91 \mathrm{GHz}$, and $116 \mathrm{GHz}$ as a function of neutral gas pressure. The data points in the plot represent the amplitude of the peak in the signals. Because the cutoff response of the waveguide is extremely sharp (typically 40 to $60 \mathrm{~dB}$ over a few $\mathrm{GHz}$ ), we interpret the sharp rise in signal amplitude as the point at which the radiation is upshifted to above the cutoff frequency. However, to compare the data with theoretical predictions, we must first determine the plasma density as a function of azulene pressure.

We have measured the plasma density with a $65 \mathrm{GHz}$ microwave interferometer in a Michelson configuration. The plasma arm of the interferometer was defined by replacing the cavity window opposite the laser input with a Teflon window. Teflon was chosen because it reflects less than quartz at this wavelength. The interferometer's microwaves then entered the cavity through the Teflon window and the portion that reflected from the quartz window at the other end interfered with the reference beam in the interferometer. We thus measured the line-averaged plasma density. The interferometer was unable to temporally resolve the rapid creation of the plasma as the ionizing laser pulse traversed the cavity, but instead recorded the slower recombination of the plasma which occurs on a $\mu$ s time scale. A typical interferometer signal is shown in Fig. 10(a). As the laser pulse traverses the cavity the interferometer signal jumps to some initial level then unwinds back to its original value as the plasma recombines. Because the plasma decays exponentially in time, the fringes are progressively farther apart for later times. By counting the number of fringes, we are able to determine the density with a good degree of accuracy. The phase change due to the presence of the plasma is related to the plasma density by

$$
\begin{aligned}
\Delta \phi_{\text {degrees }}=\frac{360}{2 \pi} L\{ & {\left[\omega_{\text {int }}^{2}-\left(\omega_{c}^{2}+\omega_{p}^{2}\right)\right]^{1 / 2} } \\
& \left.-\left[\omega_{\text {int }}^{2}-\omega_{c}^{2}\right]^{1 / 2}\right\} .
\end{aligned}
$$

$L$ is the total length of the plasma sampled by the interferometer, twice the cavity length. The frequency of the radiation used to make the measurement is $\omega_{i n t}$, and $\omega_{c}$ is the cutoff frequency of the mode of the interferometer radiation inside the cavity. The plasma density is determined from the plasma frequency, $\omega_{p}$ using (1). The measured line-averaged plasma density is plotted as a function of azulene pressure up to $16 \mathrm{mT}$ in Fig. 10(b). A least-squares fit to the data gives a conversion factor of $\sim 3.8 \times 10^{11} \mathrm{~cm}^{-3}$ per $\mathrm{mT}$ of azulene. This corresponds to a fractional ionization of $\sim 1.5 \%$ at $100^{\circ} \mathrm{C}$.

With the conversion factor from azulene pressure to plasma density the onset of the upshifted signals measured in the forward direction (see Fig. 9(b) can be plotted vs. front density as shown in Fig. 11. The horizontal bars represent the range of pressures over which the signals are sharply rising. The solid lines represent the theoretically predicted frequencies given by (3) for the forward and backward waves. The data are in reasonable agreement with the predicted linear dependence of upshift on front density. Also, the degree of upshift is approximately what is expected for the forward wave.

The detection of the backward wave is complicated by several factors. As shown in Fig. 6(a), the group velocity of the backward wave, which is initially $0.47 c$, is significantly reduced over the whole range of plasma densities obtainable in these experiments. This causes an increase in the pulse length of the backward wave, thus reducing its power, which is what we detect. Also, when the backward wave is near cutoff, its transmission coefficient at the plasma/quartz boundary is 

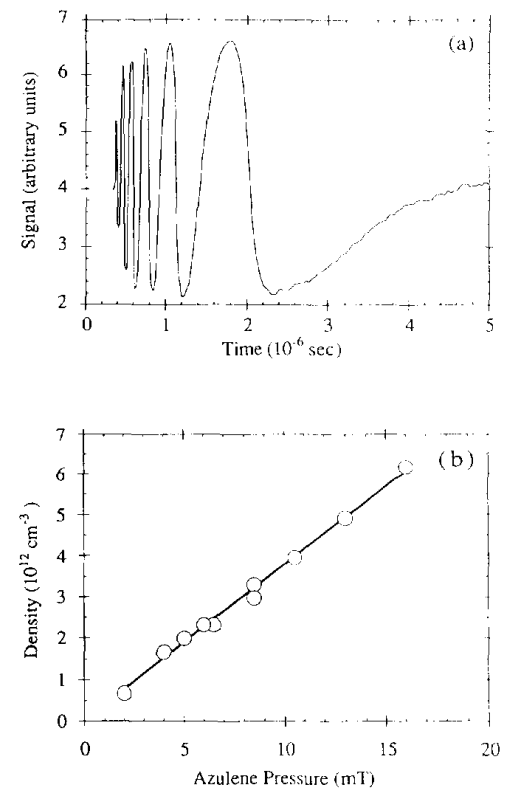

Fig. 10. (a) Typical signal from the $65 \mathrm{GHz}$ interferometer, (b) measured plasma density vs. azulene pressure for the resonant cavity configuration. The solid line is a least squares fit which gives a conversion factor of $3.8 \times 10^{11} \mathrm{~cm}^{-3}$ per mT.

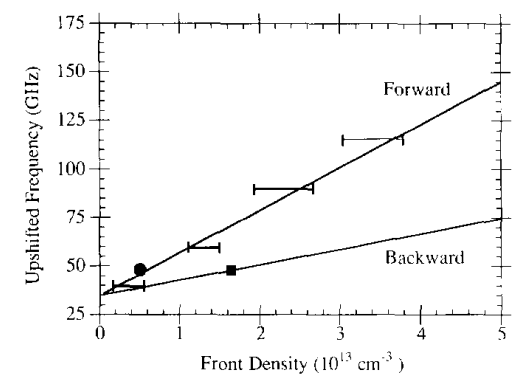

Fig. 11. Experimentally measured upshifted signal frequency vs. front density. The solid lines are the predictions of Eq. 3 .

small and the backward upshifted radiation is trapped in the cavity. The radiation detected in the backward direction has two sources: the backward wave and the forward propagating radiation that has reflected from the quartz output window into the backward direction. Because the upshifted backward wave begins to exit the cavity at the moment the ionizing laser pulse arrives, the reflected forward wave is delayed by the transit time of the laser pulse plus that of the forward radiation, $\sim 2-3 \mathrm{~ns}$. To identify the backward wave, we use a fast photo diode positioned near the entrance to the cavity to detect the arrival of the laser pulse and provide a time fiducial. We then record the temporal evolution of the radiation emitted in both the forward and backward directions as a function of azulene pressure.

Figs. 12(a) and (b) show the signals detected in the forward and backward directions on the $48 \mathrm{GHz}$ channel at an azulene pressure of $16 \mathrm{mT}$. Both signals exhibit a single subnanosecond peak and onset at approximately the same pressure, which
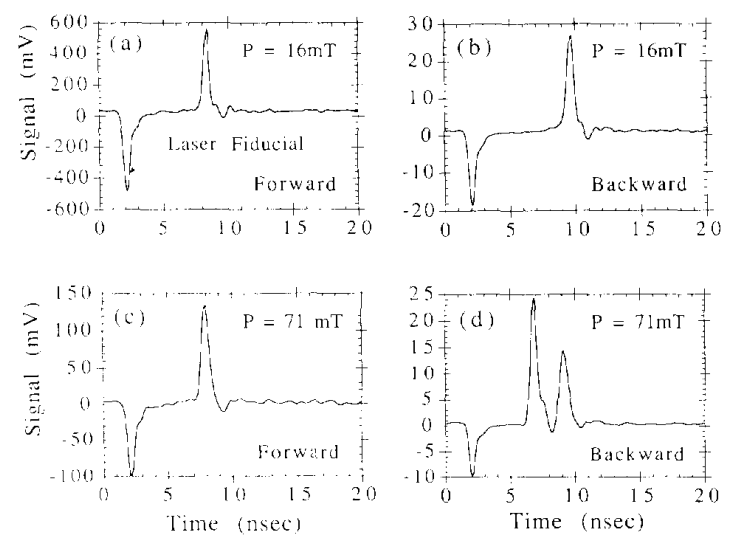

Fig. 12. Upshifted signal measured (a) in the forward direction on the $48 \mathrm{GHz}$ detector channel at $16 \mathrm{mT}$ azulene pressure, (b) in the backward direction on the $48 \mathrm{GHz}$ channel at $16 \mathrm{mT}$ pressure, (c) in the forward direction on the $48 \mathrm{GHz}$ channel at $71 \mathrm{~m}$ T pressure, (d) in the backward direction on the $48 \mathrm{GHz}$ channel at $71 \mathrm{~m}$ T pressure.

is plotted as the solid circle in Fig. 11. At this pressure, only the forward wave is expected to be upshifted above $48 \mathrm{GHz}$. We therefore interpret the backward signal as resulting from upshifted forward radiation that has reflected from the output window into the backward direction. Fig. 12(c) shows the forward signal at $70 \mathrm{mT}$ azulene pressure and Fig. 12(d) shows the backward signal at the same pressure. The forward signal continues to have only one peak, but a second earlier peak appears in the backward direction at higher pressures. The pressure at which this earlier peak onsets is shown by the solid square in Fig. 11. The onset occurs at approximately the predicted pressure for the backward wave to upshift to more than $48 \mathrm{GHz}$. This, together with the $\sim 2.4$ ns delay between the arrival of the two peaks, is consistent with identifying the earlier peak as the upshifted backward wave. However, (5) predicts that the backward wave reverses its propagation direction and exits the cavity in the forward direction when the front density is above $2 \times 10^{13} \mathrm{~cm}^{-3}$. This turn-around density corresponds to an azulene pressure of $\sim 50 \mathrm{mT}$. Above this pressure, the forward and backward radiation are expected to arrive coincidently at the forward detector. Therefore, with our present diagnostics, we are unable to differentiate between the two waves in the forward direction. That the peak associated with the backward wave persists in the backward direction at higher pressures is an issue that is investigated further in the unidirectional cavity section.

As discussed earlier, we expect the power conversion efficiency from the source wave to the upshifted wave to be $100 \%$. Remember that this does not imply that the energy conversion efficiency is also $100 \%$ because the upshifted pulse may be compressed in duration. To obtain an estimate of the power conversion efficiency, we measure the antenna pattern of the upshifted radiation in one plane (parallel to the surface of the optical table) then, assuming the output is cylindrically symmetric, integrate azimuthally to obtain the total upshifted power. As shown in Fig. 13(a), the "butterfly" antenna pattern of the $\mathrm{TE}_{01}$ mode is preserved for upshifts close to the source frequency (40 GHz channel), but becomes peaked on axis as 

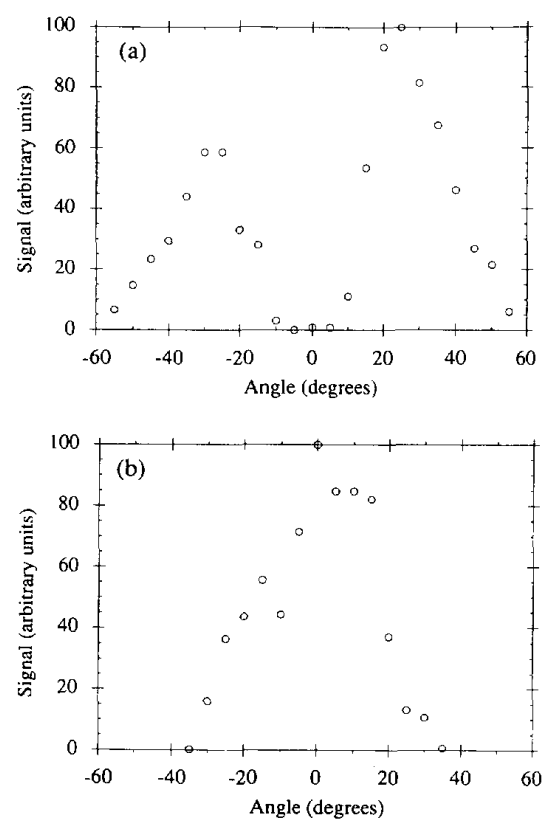

Fig. 13. Antenna pattern of the upshifted radiation measured on the (a) $40 \mathrm{GHz}$ detector channel, (b) $91 \mathrm{GHz}$ detector channel.

the degree of upshift increases, as shown in Fig. 13(b) for the $91 \mathrm{GHz}$ channel. When the laser is blocked, the total radiated power at the source frequency is approximately $5.8 \mathrm{~kW}$. The total upshifted power in the forward direction at $40 \mathrm{GHz}$ is approximately $3.1 \mathrm{~kW}$. If we define the upshifted power efficiency as upshifted power radiated in the forward direction divided by the source power radiated in the forward direction with the laser blocked, the efficiency is greater than $50 \%$ at $40 \mathrm{GHz}$. Thus defined, the efficiency is only a fraction of a percent at $90 \mathrm{GHz}$ where the forward radiated power is approximately $22 \mathrm{~W}$. This lower efficiency may be largely due to laser pump depletion along the cavity axis causing the length over which a sufficiently dense front is created to decrease. This would reduce the duration of the upshifted pulses, possibly to the point where the detector response is severely bandwidth limited. Also, we expect the response of our detection system to be significantly reduced for large upshifts where the pulsewidths are expected to be much less than $1 \mathrm{~ns}$.

\section{B. Unidirectional Microwave Cavity Setup}

In order to investigate the role of the forward and backward waves independently, we conducted a series of experiments utilizing a unidirectional waveguide configuration. This experimental arrangement is shown schematically in Fig. 14 Microwave radiation is fed into a straight section of WR19 waveguide through a $3 \mathrm{~dB}$ directional coupler $(1: 1$ power divider). The waveguide is sealed at each end with a $0.1 \mathrm{~mm}$ thick quartz window, chosen to be much less than a wavelength thick to reduce reflection. The measured ratio of the transmitted power to the reflected power is more than 10:1. The interaction waveguide consists of either one, two or three six-inch-long sections of WR-19 waveguide. It is heated, evacuated, and filled with azulene vapor as with the resonant cavity. The microwave source and the ionizing laser are also the same as those used in the previous experiments.

In the configuration shown in Fig. 14 we isolate the forward wave by launching the microwaves in the same direction as the ionizing laser. By either moving the directional coupler to the other side of the waveguide or directing the laser to enter from the other side, we are able to examine the backward wave interaction. Although we are able to launch a wave in predominantly the forward or backward direction, the upshifted radiation will leave the waveguide through a plasma/air interface. The reflections from this interface may be considerable, and we therefore expect upshifted signals to emanate from both ends of the cavity. Furthermore, small reflections from the thin quartz output window will give a small counter-propagating component to the launched wave. This may confuse the interpretation if the data when examining low amplitude upshifted signals. An additional benefit of the unidirectional waveguide over the resonant cavity is that the output is linearly polarized. This allows us to more easily utilize a diffraction grating to measure the upshifted spectra.

In previous experiments we used cutoff waveguides, acting as high-pass filters, to estimate the frequency of the upshifted radiation. This type of detection has the disadvantage that it gives little information about the spectral content of the upshifted pulse. It only senses that there is some radiation at frequencies above the cutoff frequency of the waveguide. In order to measure the spectrum of the upshifted radiation, we utilized a blazed diffraction grating with a period of $6.64 \mathrm{~mm}$. The grating is simply a rectangular aluminum slab with grooves milled into the surface. The blaze angle is $30^{\circ}$. The grating was placed approximately $2 \mathrm{~m}$ from the cavity output in the forward direction on a stage that allowed rotation about the surface of the grating as shown in Fig. 14. The angle between the incident radiation and the detected, diffracted beam was chosen to be $10^{\circ}$. This corresponds to the maximum efficiency angle for $45 \mathrm{GHz}$ radiation. The detector, as before, was a diode detector which has a known response over the frequency width of the spectrum being measured.

With the detector fixed at the $10^{\circ}$ position, we measured the spectrum of the forward upshifted radiation at several azulene pressures. These spectra are shown in Fig. 15(a). The resolution of our microwave spectrometer is shown by the detected width of the source radiation to be $\sim 2.5 \mathrm{GHz}$, Although the detector angle was fixed, rather than optimized to maintain the detector at the maximum efficiency angle, it is readily seen in Fig. 15(a) that the spectra are peaked away from the source frequency. The center frequency increases with azulene pressure, as does the width of the spectra. A more detailed, optimized measurement at an azulene pressure of $5.5 \mathrm{mT}$ is shown in Fig. 15(b). The upshifted spectrum is peaked at $\sim 46 \mathrm{GHz}$ and has a width of $\sim 10 \mathrm{GHz}$ (FWHM) without accounting for the instrument function of our microwave spectrometer.

We again measured the plasma density using a $65 \mathrm{GHz}$ microwave interferometer. In this configuration we were able to simply send the $65 \mathrm{GHz}$ radiation into the coupling arm of the directional coupler (the same port through which the source microwaves usually enter). We placed a mirror at the 


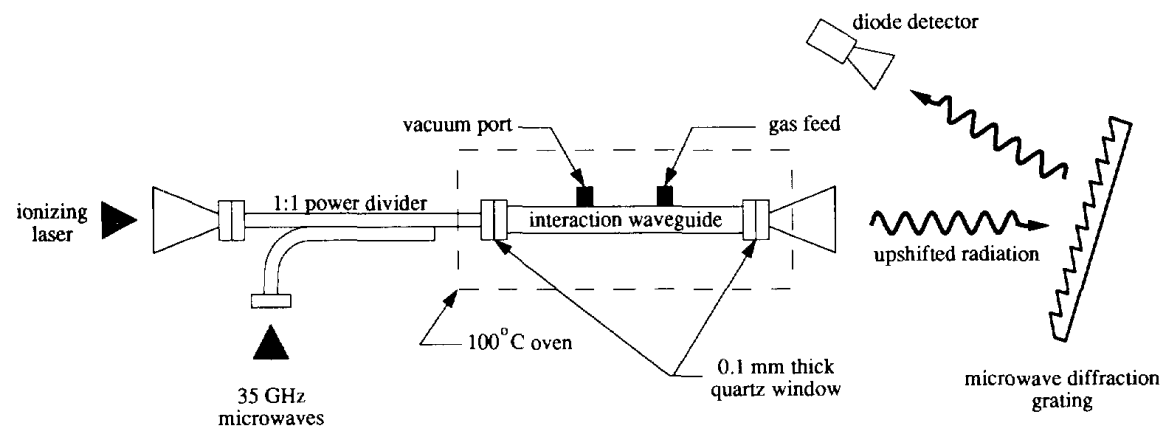

Fig. 14. Schematic of the experimental arrangement utilizing a unidirectional cavity and a diffraction grating for frequency measurements.
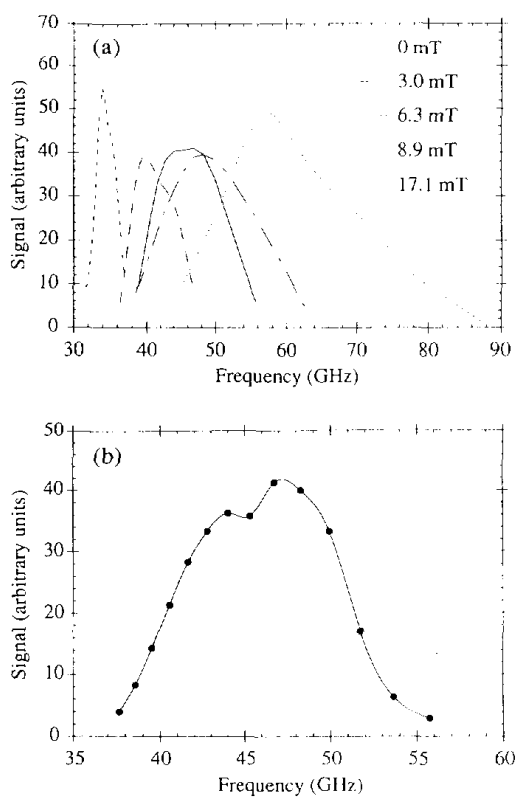

Fig. 15. (a) Spectra of upshifted forward wave radiation vs. azulene pressure (b) optimized upshifted spectrum at $5.5 \mathrm{mT}$ azulene pressure. The center frequency is $46 \mathrm{GHz}$.

output of the waveguide to reflect the microwaves back into the interferometer. A thin mica disk was taped to the center of the mirror to absorb the residual ionizing laser so that it did not make another pass through the cavity (which would cause further ionization). The measured plasma density is plotted as a function of azulene pressure in Fig. 16(a). From this measurement we obtain a conversion factor of $\sim 9 \times 10^{11} \mathrm{~cm}^{-3} / \mathrm{mT}$ of azulene. With the density measured as a function of azulene pressure, we are able to compare the upshifted frequencies with theory. This is shown in Fig. 16(b) where the error bars indicate the width (FWHM) of the upshifted spectra. These are in reasonable agreement with the theoretical predictions, and the accuracy should be able to be improved by optimizing the detector position as described earlier.

The expected pulsewidths of the pulses of upshifted radiation were derived earlier and plotted as a function of front density in Fig. 6(b). We measured the duration of the upshifted backward wave by positioning a detector preceeded by a
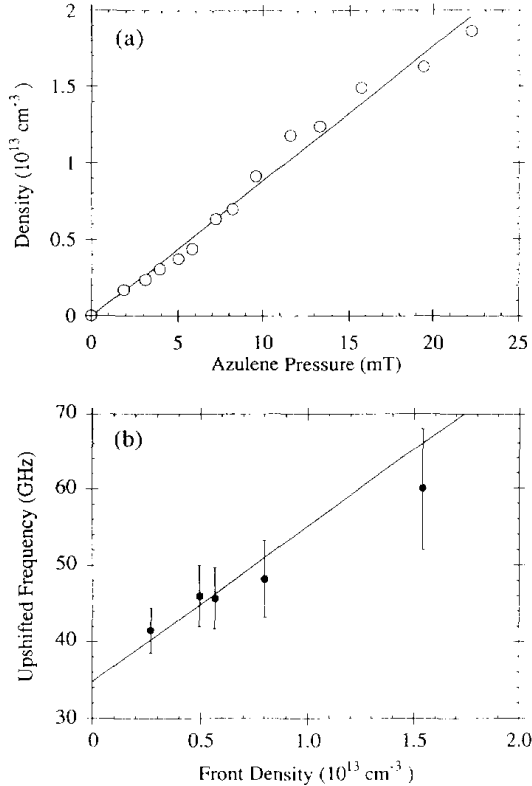

Fig. 16. (a) Plasma density measured with $65 \mathrm{GHz}$ interferometer vs, azulene pressure for the unidirectional cavity configuration. The conversion factor is $9.0 \times 10^{11} \mathrm{~cm}^{-3}$ per $\mathrm{mT}$. (b) Center frequency of upshifted spectra vs. azulene pressure. The solid line is the theoretical prediction. The error bars indicate the width (FWHM) of the upshifted spectra.

custom-made waveguide with a cutoff frequency of $36 \mathrm{GHz}$ in the backward direction. The cutoff frequency of this detector is close to the source frequency so that we can detect small upshifts. The source frequency rejection is sufficiently high that we are unable to detect a signal from the source wave when we fire the magnetron with the ionizing laser blocked. Fig. 17(a) shows a backward upshifted signal recorded on the $36 \mathrm{GHz}$ detector in the backward direction at an azulene pressure of $4 \mathrm{mT}$. The pulsewidth is $\sim 7.5 \mathrm{~ns}$. Fig. 17(b) is a similar signal taken with a $116 \mathrm{GHz}$ cutoff detector in the forward direction while launching a forward wave. The pulsewidth is detection limited to $\sim 0.5 \mathrm{nsec}$.

In Fig. 18(a) we plot upshifted pulsewidths measured in the forward and backward directions as a function of plasma density. The solid curves are the predictions of (50). At low azulene pressures, $2-8 \mathrm{mT}$, the backward signals are flat-top and long, 7-8 nsec, as expected. At pressures above $\sim 10 \mathrm{mT}$, 

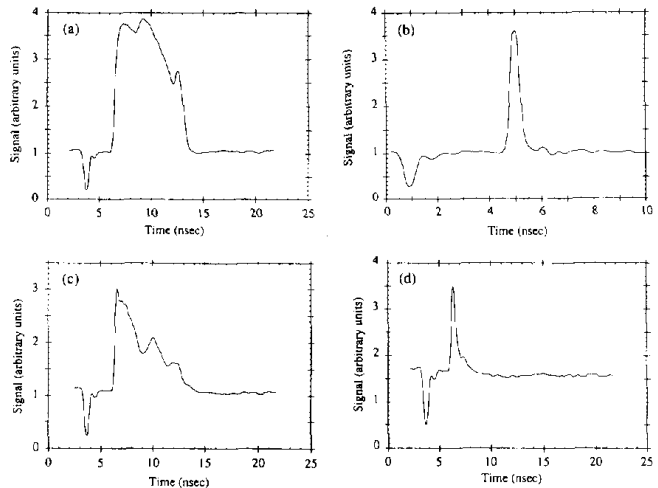

Fig. 17. (a) Backward upshifted signal recorded with a $36 \mathrm{GHz}$ cutoff detector in the backward direction at $4 \mathrm{mT}$ azulene pressure. The pulsewidth is 7.5 ns. (b) Forward upshifted signal recorded with a $116 \mathrm{GHz}$ cutoff detector in the forward direction at $49 \mathrm{mT}$ pressure. The pulsewidth is detector-limited to $0.5 \mathrm{~ns}$. (c) Backward upshifted signal recorded with a $36 \mathrm{GHz}$ cutoff detector in the backward direction at $10 \mathrm{mT}$ pressure. The trailing edge of the pulse is partially eroded at this pressure. (d) Backward upshifted signal recorded with a $36 \mathrm{GHz}$ cutoff detector in the backward direction at $16 \mathrm{mT}$ pressure. The trailing edge of the pulse has decayed leaving a 1 ns peak at the leading edge.

the trailing edges of the flat-top pulses begin to erode, leaving a narrow peak at the leading edge of the pulse. Fig. 17(c) shows a partially eroded backward pulse at $10 \mathrm{mT}$ of azulene. At $16 \mathrm{mT}$ the long flat-top pulse has almost completely decayed, leaving a 1-nsec peak at the leading edge (see Fig. 17(d)). This decay may be a manifestation of the group velocity of the upshifted backward wave approaching zero as it nears cutoff. At higher pressures, because we expected the backward wave to have turned around, we positioned a higher frequency detector in the forward direction. The observed pulsewidths, shown in Fig. 18(a) (open circles in the $3-8 \times 10^{13} \mathrm{~cm}^{-3}$ density range), are significantly shorter than expected for the "reflected" backward wave, but longer than the duration of the forward pulses at the same pressures (open squares in the figure). We expected to see long pulses in the forward direction when the backward wave had turned around but not yet attained a high group velocity in the forward direction. Such pulses were not observed in the forward direction. However, at pressures above which the backward wave is expected turn around, the observed pulsewidths are consistently longer for the backward wave than for the forward wave. This is consistent with what we would expect for the "reflected" backward wave.

The durations of the upshifted forward pulses, shown by the open squares in Fig. 18(a), agree reasonably well with the theoretical predictions. This can be seen more clearly in Fig. 18(b), a detail of Fig. 18(a) showing the detectors used to make the measurements. At plasma densities above about $3 \times 10^{-3} \mathrm{~cm}^{-3}$ the pulsewidths are detection limited to $\sim 0.5$ nsec. These pulses should continue to compress in duration as the group velocity of the radiation increases at higher front densities.

\section{CONCLUSIONS}

We have demonstrated that a relativistically propagating, underdense ionization front can significantly upshift the fre-
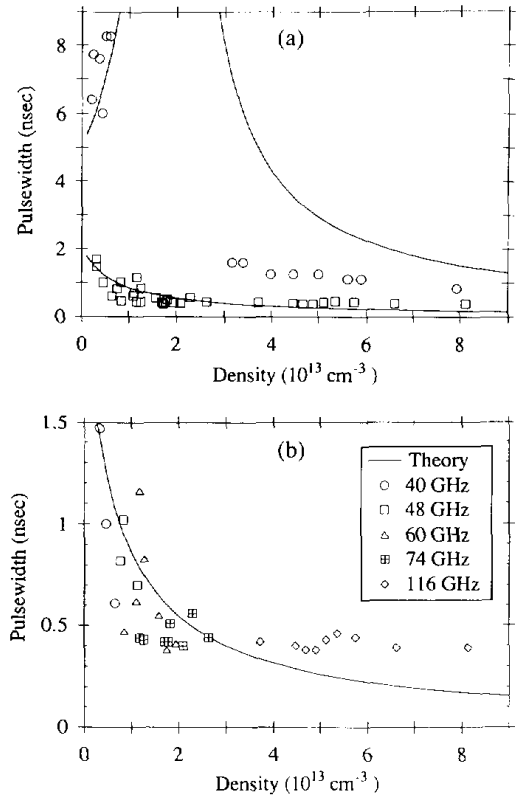

Fig. 18. (a) Measured upshifted pulsewidth of the forward (open squares) and backward (open circles) waves vs. ionization front denisty. The solid curves are the theoretical predicitons of Eq. 50. (b) Detail of the pulsewidth plot for the forward wave.

quency of an impinging electromagnetic wave. The degree of upshift, which is proportional to the plasma density in the front, has been turned continuously from $35 \mathrm{GHz}$ to more than $173 \mathrm{GHz}$ by varying the neutral gas pressure in the microwave cavity. The forward-propagating radiation is upshifted by a larger amount than the backward-propagating radiation for a given plasma density, and subnanosecond upshifted pulses were observed. These results are in good agreement with theoretical predictions.

We observed the expected compression of the duration of the forward wave with increasing plasma density. We also saw the duration of the backward wave increase as its group velocity initially decreased with increasing plasma density. Although we observed the erosion of the tail of the backward upshifted pulses near the preducted turn-around density, we were unable to definitively conclude that the backward wave had "reflected" into the forward direction. At high plasma densities we observed high frequency, short pulses while launching the backward wave and their duration was greater than that observed while launching the forward wave at the same pressures, as expected for the backward wave "reflection." However, we were unable to determine their initial direction of propagation inside the waveguide. The observation of this "reflected" wave will undoubtedly be the emphasis of future experiments.

While there is no fundamental reason why this technique could not be applied just as well to other frequency regimes, present technological constraints on the number of ionizing photons that can be contained in a short pulse make the high frequency microwave regime most appropriate. To obtain large upshifts at lower frequencies the volume containing the source wave would be too large to ionize to high plasma density and at 
higher frequencies the required plasma density is prohibitively large. We have considered upshifting visible pulses inside a high pressure gas jet by focusing our ionizing laser. This may prove feasible for ultra-short pulse generation with modest upshifts, but very large upshifts would require close to solid density plasmas.

This work represents only the first set of experiments with underdense ionization fronts. There are many related experiments still to be undertaken. These include continuation of the effort to observe the "reflection" of the backward wave and observation of the static magnetic field associated with the free-streaming mode. Furthermore, plasma physics allows many interesting variations on these basic experiments, including operation with different waveguide modes and the application of an external magnetic field. Some of these are presently being considered theoretically [22] and will surely be included in future experiments.

\section{ACKNOWLEDGMENT}

The authors wish to thank Gennady Shvets, Don Hopkins, and Drs. Tudor Johnston, Andy Sessler, and Tom Katsouleas for their invaluable assistance with these experiments.

\section{REFERENCES}

[1] J.D. Jackson, Classical Electrodynamics (2nd ed.). New York: Wiley, 1975 , p. 521.

[2] V.I. Semenova, "Reflection of electromagnetic waves from an ionization front," Sov. Radiophys. and Quantum Electron., vol. 10, pp. 599-604, 1967.

[3] M. Lampe, E. Ott, and J.H. Walker, "Interaction of electromagnetic waves with a moving ionization front," Phys. Fluids, vol. 10, pp. 42-54, 1978.

[4] W.B. Mori, "Generation of tunable radiation using an underdense ionization front," Phys. Rev. A, vol. 44, pp. 5118-5121, 1991.

[5] E. Yablonavitch, "Self-phase modulation of light in laser-breakdown plasma," Phys. Rev. Lett., vol. 32, pp. 1101-1104, 1974.

[6] P. B. Corkum, "High-power, subpicosecond $10-\mu$ m pulse generation," Opt. Lett., vol. 8, pp. 514-516, 1983.

[7] W.M. Wood, C. W. Siders, and M. C. Downer, "Measurement of femtosecond ionization dynamics of atmospheric density gases by spectral blueshifting," Phys. Rev. Lett., vol. 67, pp. 3523-3526, 1991.

[8] S.C. Wilks, J.M. Dawson, W.B. Mori, T.C. Katsouleas, and M.E. Jones, "Photon accelerator," Phys. Rev. Lett., vol. 62, pp. 2600-2603, 1989.

[9] E. Esarey, G. Joyce, and P. Sprangle, "Frequency up-shifting of laser pulses by copropagating ionization fronts," Phys. Rev. A, vol. 44, pp. 3908-3911, 1991.

[10] P. Sprangle, E. Esarey, and A. Ting, "Nonlinear interaction of intense laser pulses in plasmas," Phys. Rev. A, vol. 41, pp. 4463-4469, 1990.

[11] R. L. Savage Jr., W. B. Mori, C. Joshi, T. W. Johnston, and G. Shvets, "Tunable radiation generation using underdense ionization fronts," International Conference on Research Trends in Coherent Radiation Generation and Particle Accelerators (La Jolla, CA), Feb. 11-13, 1991.

[12] R. L. Savage Jr., C. Joshi, and W. B. Mori, "Generation of highly tunable microwave radiation via a relativistic ionization front," Proc. IEEE MTT-S Int. Microwave Symp. (Boston, MA), 1991.

[13] R. L. Savage Jr., C. Joshi, and W. B. Mori, "Frequency upconversion of electromagnetic radiation upon transmission into an ionization front," Phys. Rev. Lett., vol. 68, pp. 946-949, 1992.

[14] H.C. Kapteyn and M.M. Murnane, "Relativistic pulse compression," J. Opt. Soc. Am. B, vol. 8, pp. 1657-1662, 1991.

[15] S. C. Wilks, J. M. Dawson, and W. B. Mori, "Frequency up-conversion of electromegnetic radiation with use of an overdense plasma," Phys. Rev. Lett., vol. 61, pp. 337-340, 1988.

[16] C. J. Joshi, C.E. Clayton, K. Marsh, D. B. Hopkins, and A. Sessler "Demonstration of frequency upshifting of microwave radiation by rapid plasma creation," IEEE Trans. Plasma Sci., vol. 18, pp. 814-818, 1990
[17] S.P. Kuo, "Frequency up-conversion of microwave pulse in a rapidly growing plasma," Phys. Rev. Lett., vol. 65, pp. 1000-1003, 1990.

[18] V. B. Gil'denburg, A. V. Kim, and A. M. Sergeev, "Possibility of sharp increase in the frequency of the radiation of ionizing laser pulse in gas," JETP Lett., vol. 51, pp. 104-107, 1990.

[19] D. Kalluri and R. K. Shrivastava, "Electromagnetic wave interaction with moving bounded plasmas," J. Appl. Phys., vol. 44, pp. 4518-4521, 1973.

[20] R. L. Savage Jr., R.P. Brogle, W. B. Mori, and C. Joshi, "Photon acceleration via laser-produced ionization fronts." Eighth International Conference on Ultrafast Phenomena (Antibes-Juan les Pins, France), June 8-12, 1992.

[21] S. Ramo, J.R. Whinnery, and T. Van Duzer, Fields and Waves in Communication Electronics (2nd ed.). New York: Wiley, 1984, p. 425

[22] C. H. Lai, T. C. Katsouleas, W. B. Mori, and D. Whittim, "Frequency upshifting by an ionization front in a magnetized plasma," IEEE Trans. Plasma Sci, vol. 21, Feb. 1993.

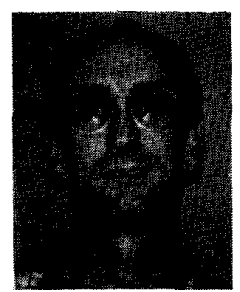

Richard L. Savage, Jr. was born in Enid, OK in 1954. He received the B.S. degree in physics from UCLA in 1976. After he worked several years on far-infrared lasers for fusion plasma diagnostics, he returned to graduate school in the Electrical Engineering Department at UCLA, where he received the M.S. degree in 1988 and the Ph.D. degree in 1992.

He is currently employed as a staff physicist by the Physics, Math, and Astronomy departments at the California Institute of Technology, where he is working on the Laser Interferometer Gravitational-Wave Observatory (LIGO) project.

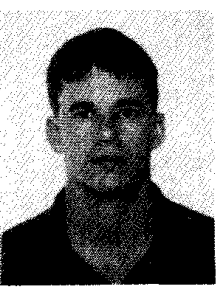

Robert P. Brogle was born in Gainesville, FL in 1967. He received the B.S. degree in physics from Stanford University. He is currently enrolled in the $\mathrm{Ph} . \mathrm{D}$. program in the Physics Department at UCLA.

Warren B. Mori, for a photograph and biography, please see page 4 of this issue.

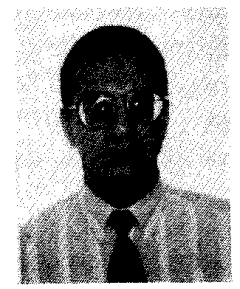

Chan Joshi was born in India in 1953. He teceived the B.S. degree in nuclear engineering from London University in 1974. He received the Ph.D. degree in applied physics in 1978 from Hull University, England.

He held a postdoctoral position at the National Research Council in Ottawa, Canada for 2 years. Since 1980 he has been with the Electrical Engineering Department at UCLA, first as a member of the research staff and currently as Full Professor He teaches both undergraduate and graduate courses in electromagnetics and quantum electronics. He has published over 100 technical papers. His research interests are in the areas of collective particle accelerators, laser-plasma interactions/nonlinear optics, development of Xray CCD's and ultrahigh power lasers.

Dr. Joshi is a Fellow of IEEE and APS. 